\title{
The Hell Lyman alpha forest and the thermal state of the IGM
}

\author{
C. Fechner and D. Reimers
}

\author{
Hamburger Sternwarte, Universität Hamburg, Gojenbergsweg 112, 21029 Hamburg, Germany \\ e-mail: [cfechner,dreimers]@hs.uni-hamburg.de
}

Received 13 October 2006 / Accepted 15 November 2006

\begin{abstract}
Aims. Recent analyses of the intergalactic UV background by means of the He II Ly $\alpha$ forest assume that He II and H I absorption features have the same line widths. We omit this assumption to investigate possible effects of thermal line broadening on the inferred $\mathrm{He}$ II/H I ratio $\eta$ and to explore the potential of intergalactic He II observations to constrain the thermal state of the intergalactic medium.

Methods. Deriving a simple relation between the column density and the temperature of an absorber based on the temperature-density relation $T=T_{0}(1+\delta)^{\gamma-1}$ we develop a procedure to fit $T_{0}, \gamma$, and $\eta$ simultaneously by modeling the observed spectra with Doppler profiles. In an alternative approach the temperature $T$ of an absorber, the He II/H I ratio $\eta$, and the redshift scale of $\eta$ variations are estimated simultaneously by optimizing the Doppler parameters of He II.

Results. Testing our procedure with artificial data shows that well-constrained results can be obtained only if the signal-to-noise ratio in the $\mathrm{He}$ II forest is $S / N \gtrsim 20$. Additionally, ambiguities in the line profile decomposition may result in significant systematic errors. Thus, it is impossible to give an estimate of the temperature-density relation with the He II data available at present $(S / N \sim 5)$. However, we find that only $45 \%$ of the lines in our sample favor turbulent line widths while the remaining lines are probably affected by thermal broadening. Furthermore, the inferred $\eta$ values are on average about 0.05 dex larger if a thermal component is taken into account, and their distribution is $46 \%$ narrower in comparison to a purely turbulent fit. Therefore, variations of $\eta$ on a $10 \%$ level may be related to the presence of thermal line broadening. The apparent correlation between the strength of the H I absorption and the $\eta$ value, which has been found in former studies assuming turbulent line broadening, essentially disappears if thermal broadening is taken into account. In the redshift range $2.58<z<2.74$ towards the quasars HE 2347-4342 and HS 1700+6416 we obtain $\eta \approx 100$ and slightly larger. In the same redshift range the far-UV spectrum of HS $1700+6416$ is best and we estimate a mean value of $\log \eta=2.11 \pm 0.32$ taking into account combined thermal and turbulent broadening.
\end{abstract}

Key words. cosmology: observations - quasars: absorption lines - quasars: individual: HE 2347-4342 quasars: individual: HS 1700+6416 - intergalactic medium

\section{Introduction}

The temperature of the intergalactic medium (IGM) preserves a memory of the reionization history of the universe (e.g. Theuns et al. 2002a) and provides constraints on the nature of the reionizing sources (e.g. Tittley \& Meiksin 2006). The usual approach to measure the temperature considers the Doppler parameter distribution of Ly $\alpha$ forest absorbers (e.g. Schaye et al. 1999, 2000; Ricotti et al. 2000; Bryan \& Machacek 2000; McDonald et al. 2001; but see Theuns et al. 2002b, for a different approach). Generally, there is a power law dependence between the column density $N_{\mathrm{HI}}$ and the lowest Doppler parameter at this column density, i.e. the cut-off $b_{\min }\left(N_{\mathrm{HI}}\right)$. Interpreting $b_{\min }$ as purely thermal, the temperature of the IGM can be estimated. In practice, hydrodynamic simulations are required to transform the $b_{\min }-N_{\mathrm{H} \text { I }}$ dependency into a temperature-density relation (e.g. Schaye et al. 1999). However, even for the narrowest lines the thermal component is not necessarily the only contribution to the line width. Thus, the cut-off provides just an upper limit of the temperature. Further broadening is introduced by the velocity field including peculiar differential motions and the Hubble flow. The observed distribution of $b$-parameters is also related to the amplitude of the primordial density fluctuations as shown by Hui \& Rutledge (1999) using linear perturbation theory.
The approach of estimating the cut-off of the $b\left(N_{\mathrm{HI}}\right)$ distribution to measure the temperature of the IGM is based on the concept of a well-defined power law relation between temperature and density. Hui \& Gnedin (1997) showed this relation to be valid in the low-density Ly $\alpha$ forest for uniform reionization models as a consequence of the interplay of photoionization heating and adiabatic cooling due to the cosmic expansion. Valageas et al. (2002) argued that the power law relation is still valid even if the ionizing UV background is inhomogeneous as long as the gas is in local ionization equilibrium. The relation breaks at high densities since in the non-linear regime shockheating due to gravitational processes becomes dominant introducing a second IGM component, the warm-hot intergalactic medium (WHIM) containing the bulk of the baryons at the present epoch (e.g. Cen \& Ostriker 1999; Davé et al. 2001; Valageas et al. 2002; Kang et al. 2005). On the other hand, highdensity gas may cool down in less than a Hubble time which is a precondition to enable star formation. Thus, cool dense gas exists at $z \sim 2-3$ and even at lower redshifts (e.g. Davé et al. 1999; Valageas et al. 2002). As a consequence the temperature-density relation breaks at high densities $\left(\delta \sim 10\right.$ or $N_{\mathrm{HI}} \sim 10^{15} \mathrm{~cm}^{-2}$, respectively). Above this threshold the gas temperature decreases with density (for a summary of the phases of the IGM see e.g. Fig. 1 of Valageas et al. 2002). 
In agreement with the theoretical scenario Misawa et al. (2004) observed, indeed, that due to shielding effects high density lines are narrower and therefore colder than low density absorbers. Whereas Rauch et al. (2005) found from an investigation of the velocity structure of Ly $\alpha$ forest clouds in the spectra of multiple QSO sight lines that the large scale motions in the IGM are dominated by the Hubble flow. According to Rauch et al. (2005) some absorbers undergo early stages of gravitational collapse but the majority of absorbers apparently arise from expanding filaments accelerated by the infall into more massive structures.

In a study of the reionization of helium Gleser et al. (2005) find that the temperature-density relation may trace the stages of He II reionization. With ongoing reionization the slope of the temperature-density relation gets steeper and a significant scatter is introduced. At $z \sim 2-3$ the scatter affects in particular the low density regime. When helium reionization is completed the slope of the power law flattens again and the amount of scatter decreases since adiabatic cooling dominates over photoionization heating. Similar results have been obtained by Bolton et al. (2004) who examined the effects of radiative transfer on the temperature-density relation. Indeed, the mean temperature of the IGM peaks at $z \sim 3$ (Schaye et al. 2000; Theuns et al. $2002 \mathrm{a}, \mathrm{b})$ which is thought to be due to additional heating because of He II reionization.

From the analysis of the resolved He II Ly $\alpha$ forest in the spectra of the quasars HE 2347-4342 and HS $1700+6416$ a correlation between the density of the absorbers and the hardness of the ionizing radiation (represented by the parameter $\eta$ which is the $\mathrm{He}$ II/H I column density ratio) has been found in the sense that higher column density absorbers are apparently exposed to a harder radiation field (Shull et al. 2004; Zheng et al. 2004; Reimers et al. 2006; Fechner et al. 2006a). There are several suggestions for a physical explanation of this correlation like self-shielding and shadowing effects (Maselli \& Ferrara 2005; Tittley \& Meiksin 2006) and the spatial distribution of QSOs as the sources of hard radiation (Bolton et al. 2006). However, Fechner \& Reimers (2007) suggested that part of the correlation might be due to the unjustified assumption that the line widths of the absorption features are dominated by purely turbulent broadening. If this assumption is incorrect, a correlation between the $\eta$ value and the absorber density will be introduced artificially. In order to reproduce a given equivalent width of an absorption line the column density will be overestimated if the line width is underestimated. This effect is in particular pronounced for strong absorption features with high column densities that are beyond the linear part of the curve of growth and thus introduce an apparent correlation between $\eta$ and H I column density.

Measurements of observed features indicate that the lines are indeed turbulently broadened (Zheng et al. 2004). However, this result is based on a limited statistical sample of 8 absorbers. Liu et al. (2006) claimed to confirm this finding using hydrodynamic simulations. On the other hand, Fechner \& Reimers (2007) showed that statistically, lines with apparent low $\eta$ values are often dominated by thermal broadening. In order to solve this apparent conflict further investigation is needed.

In this work we introduce a simple approach to consider the temperature of the IGM when analyzing the He II Ly $\alpha$ forest. This provides, at least in principle, an independent method to measure the temperature-density relation (often referred to as equation of state of the intergalactic medium). Since HE 2347-4342 and HS $1700+6416$ are the only objects where high resolution FUSE spectra are available (Kriss et al. 2001; Shull et al. 2004; Zheng et al. 2004; Fechner et al. 2006a), we apply our method to these sight lines. However, as we will see, the data quality $(S / N \sim 5)$ is insufficient to derive tight constraints for the temperature-density relation.

After shortly reviewing several equations important in the context of the temperature-density relation in Sect. 2, Sect. 3 presents the methodical approach to derive the parameters of the temperature-density relation and $\eta$ from observations of the He II Ly $\alpha$ forest in comparison to H I. This method is tested using artificial data in Sect. 4 and applied to the observations in Sect. 5. In Sect. 6 we introduce an alternative approach to estimate the temperature for the individual absorption lines more directly and discuss the effect on the inferred $\eta$ values.

\section{The thermal state of the IGM}

Hui \& Gnedin (1997) have shown that there is a well-defined power law relation between temperature and density, often referred to as equation of state for the low density IGM $(\delta \lesssim 10$, i.e. $N_{\mathrm{HI}} \lesssim 10^{15} \mathrm{~cm}^{-2}$ ),

$T=T_{0}(1+\delta)^{\gamma-1}$,

where $\delta=\frac{\rho}{\bar{\rho}}-1$ is the gas overdensity and $T_{0}$ the temperature at the mean density. This equation of state can be converted into a power law relation between the cut-off Doppler parameter $b_{\text {min }}$ and the $\mathrm{H}$ I column density using cosmological simulations (Schaye et al. 1999). Therefore, estimates of the parameters $T_{0}$ and $\gamma$ are usually based on measuring the cut-off of the $b\left(N_{\mathrm{HI}}\right)$ distribution. The combination of $\mathrm{H} \mathrm{I}$ and He II data would provide, at least in principle, an independent estimate of the thermal state of the IGM. In order to relate the equation of state to the observed $\mathrm{HI}$ and He II spectra it is necessary to connect the H I column density $N_{\mathrm{HI}}$ and the temperature of the absorbing material.

Assuming that the almost fully ionized gas is in photoionization equilibrium, the number density of neutral hydrogen can be written as

$$
\begin{aligned}
n_{\mathrm{HI}} \approx & 1.41 \times 10^{-14} \mathrm{~cm}^{-3}(1+\delta)^{2}(1+z)^{6}\left(\frac{T}{10^{4} \mathrm{~K}}\right)^{-0.755} \\
& \times\left(\frac{\Gamma_{\mathrm{HI}}}{10^{-12} \mathrm{~s}^{-1}}\right)^{-1}\left(\frac{\Omega_{\mathrm{b}} h^{2}}{0.02}\right)^{2}
\end{aligned}
$$

with the photoionization rate of hydrogen $\Gamma_{\mathrm{HI}}$. In order to derive this relation we have used

$n_{\mathrm{H}}=\frac{3 \Omega_{\mathrm{b}} H_{0}^{2}}{8 \pi G m_{\mathrm{H}}}(1-Y)(1+z)^{3}(1+\delta)$,

with the primordial mass fraction of helium $Y \approx 0.244$ (Burles et al. 2001).

Since $N_{\mathrm{HI}}=n_{\mathrm{HI}} \cdot l$, Eq. (2) leads to a relation between H I column density and overdensity $\delta$. However, the length $l$ of the absorbers has to be known. Bryan \& Machacek (2000) choose a typical size of absorbers from simulations scaling with $l \propto(1+z)^{-1}$. Schaye (2001) argued that the characteristic size of an absorber along any line of sight will generally be in order of the local Jeans length

$$
L_{\mathrm{J}} \approx \sqrt{3.59 \times 10^{42} \mathrm{~cm}^{-1}\left(\frac{T}{10^{4} \mathrm{~K}}\right)\left(\frac{f_{\mathrm{g}}}{0.16}\right) n_{\mathrm{H}}^{-1}},
$$

with the fraction $f_{\mathrm{g}}$ of mass in the gas which should be close to the universal value $f_{\mathrm{g}} \approx \Omega_{\mathrm{b}} / \Omega_{\mathrm{m}} \sim 0.16$ in the low density 
Ly $\alpha$ forest. Combining Eqs. (2)-(4) leads to an analytic expression for the relation between the observable column density of neutral hydrogen $N_{\mathrm{HI}}$ and the corresponding overdensity (see also Schaye 2001)

$$
\begin{aligned}
& N_{\mathrm{HI}} \approx 6.50 \times 10^{10} \mathrm{~cm}^{-2}(1+\delta)^{1.5}(1+z)^{4.5}\left(\frac{T}{10^{4} \mathrm{~K}}\right)^{-0.255} \\
& \times\left(\frac{\Gamma_{\mathrm{HI}}}{10^{-12} \mathrm{~s}^{-1}}\right)^{-1}\left(\frac{\Omega_{\mathrm{b}} h^{2}}{0.02}\right)^{1.5}\left(\frac{f_{\mathrm{g}}}{0.16}\right)^{0.5} .
\end{aligned}
$$

The latter three parameters are in the order of unity. Then, inserting the equation of state yields

$$
\begin{aligned}
\log N_{\mathrm{H}_{\mathrm{I}} \approx} & 11.833+4.5 \cdot \log (1+z)-\frac{1.5}{\gamma-1} \log T_{0} \\
& +\left(\frac{1.5}{\gamma-1}-0.255\right) \cdot \log T,
\end{aligned}
$$

where $N_{\mathrm{HI}}$ is measured in $\mathrm{cm}^{-2}$ and the temperatures are given in $\mathrm{K}$. Thus, we have found a relation between the H I column density and the temperature of an absorber which only depends on the redshift and the parameters of the equation of state $\gamma$ and $\log T_{0}$. However, its validity is limited to gas with low and moderate densities. At higher densities $\left(N_{\mathrm{HI}} \gtrsim 10^{15} \mathrm{~cm}^{-2}\right)$ radiative cooling dominates over adiabatic cooling due to the Hubble expansion, leading to lower temperatures. Furthermore, a possible scatter in the temperature-density relation due to helium reionization (Gleser et al. 2005) is neglected.

\section{Method}

Three different analysis methods have been applied to the observations of the He II Ly $\alpha$ forest so far: line profile fitting (Kriss et al. 2001; Zheng et al. 2004; Fechner et al. 2006a), an apparent optical depth method (Shull et al. 2004; Fechner et al. 2006a) and spectrum fitting (Fechner \& Reimers 2007). The latter fits the high-quality optical H I spectrum directly to the He II data, and allows to estimate the length scales of $\eta$ variations. Among these three methods only profile fitting provides the possibility of taking into account different line widths of $\mathrm{HI}$ and $\mathrm{He}$ II as would be introduced by thermal line broadening. In order to preserve the advantage of the spectrum fitting method to estimate scales on which the ionizing background fluctuates, we combine the traditional profile fit analysis and the spectrum fitting method.

The H I Ly $\alpha$ forest is fitted with Doppler profiles. From the resulting line list an artificial He II spectrum is computed and convolved with an appropriate instrumental profile. The artificial spectrum can then be fitted to the FUSE data by scaling the H I column density. Furthermore, the line width of the He II features can be addressed via the Doppler parameter $b_{\text {He II }}$ which could lead in principle to an independent estimate of the temperature of the IGM and the parameters of the equation of state. As we will see below, fitting the temperature is sensitive to the noise level of the He II spectrum. The quality of present day He II data from FUSE is insufficient to derive well constrained temperatures and scales of variation for $\eta$ simultaneously. Consequently, we will a priori select redshift ranges where $\eta$ is assumed to be constant.

Fitting line profiles introduces further uncertainties. As demonstrated by Fechner et al. (2006a), a scatter of 10-15\% in $\eta$ is expected even if the underlying value is constant. This is due to the fact that any line profile model recovers the observed data only imperfectly. Additionally, the high noise level of the FUSE data introduces further uncertainties (see also Liu et al. 2006). Since these problems are inevitable, we expect that our results can be interpreted statistically but conclusions for single absorbers should be considered very carefully.

The modified spectrum fitting method works as follows: first, the H I data is fitted and a line list with the values of redshift $z$, column density $\log N_{\mathrm{HI}}$, and Doppler parameter $b_{\mathrm{HI}}$ is prepared. Then values of the parameters $\log T_{0}$ and $\gamma$ are selected. The temperature $T$ of each single line is computed according to Eq. (6). The Doppler parameter of He II is then given by

$b_{\mathrm{He} \text { II }}=\sqrt{b_{\mathrm{HI}}^{2}-2 k T \cdot\left(\frac{1}{m_{\mathrm{H}}}-\frac{1}{m_{\mathrm{He}}}\right)}$,

where $k$ is Boltzmann's constant and $m_{\mathrm{H}}$ and $m_{\mathrm{He}}$ are the masses of the hydrogen or helium atom, respectively. If the temperature is very high, the term beneath the square-root in Eq. (7) may become negative. In this case the Doppler parameter of $\mathrm{HI}$ is assumed to be completely thermal and the temperature is set to $T=b_{\mathrm{HI}}^{2} \cdot m_{\mathrm{H}} /(2 k)$. From the adjusted line parameters an artificial He II spectrum is generated and fitted to the data using a $\chi^{2}$-procedure where $\log \eta$ is varied (as described in Fechner \& Reimers 2007). This means, we assume $\eta$ to be constant in the considered redshift range. This procedure is repeated for several values of $\log T_{0}$ and $\gamma$. We explore the parameter space $3.50 \leq$ $\log T_{0} \leq 4.50$ and $0.70 \leq \gamma \leq 1.80$ where realistic results are expected. By comparing the resulting $\chi^{2}$-values for each combination of $\log T_{0}$ and $\gamma$ the best fit parameters can be found. In total three free parameters $\left(\log \eta, \log T_{0}\right.$, and $\left.\gamma\right)$ are estimated for a given redshift interval.

Equation (7) assumes that the thermal and turbulent Doppler parameters both lead to a Gaussian profile and combine to the

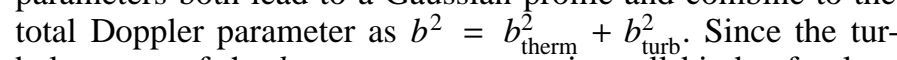
bulent part of the $b$-parameter summarizes all kinds of velocities except thermal, the broadening profile is not necessarily Gaussian. In particular, due to the atomic parameters of helium, absorbers with moderate He II column densities probe the low density $\operatorname{Ly} \alpha$ forest and are probably dominated by the differential Hubble flow rather than microscopic turbulence. Thus, Eq. (7) represents the most simple approach to combine thermal and turbulent line broadening. This should be sufficient for a first analysis.

\section{Application to artificial data}

In order to explore the potential of the introduced approach we examine artificial data which is created on the basis of the statistical properties of the $\operatorname{Ly} \alpha$ forest. The column density distribution function with $\beta=1.5$ is adopted from Kirkman \& Tytler (1997). Values in the range $11.0 \leq \log N_{\mathrm{HI}} \leq 18.0$ are simulated. The Doppler parameter distribution is described by a truncated Gaussian $\left(b_{\min }=10 \mathrm{~km} \mathrm{~s}^{-1}\right)$ centered at $27 \mathrm{~km} \mathrm{~s}^{-1}$ with a with of $\sigma_{b}=8.75 \mathrm{~km} \mathrm{~s}^{-1}$ in agreement with $\mathrm{Hu}$ et al. (1995). In addition the temperature is linked to the H I column density according to Eq. (6). The parameters of the temperature-density relation are set to the realistic values $\log T_{0}=4.3$ and $\gamma=1.3$ (e.g. Schaye et al. 2000). Furthermore, we chose $\log \eta=1.903$ (i.e. $\eta=80$; e.g. Kriss et al. 2001). The spectra cover the redshift range $2.56<z<2.92$ with $R=40000$ in the $\mathrm{HI}$ and $R=15000$ in the He II Ly $\alpha$ forest. In order to study the importance of the data quality several signal-to-noise ratios are chosen for the artificial He II spectrum $(S / N=5,10,20,30,50,100)$. The $S / N$ for the H I Ly $\alpha$ forest is set to 100 which is comparable to the noise 


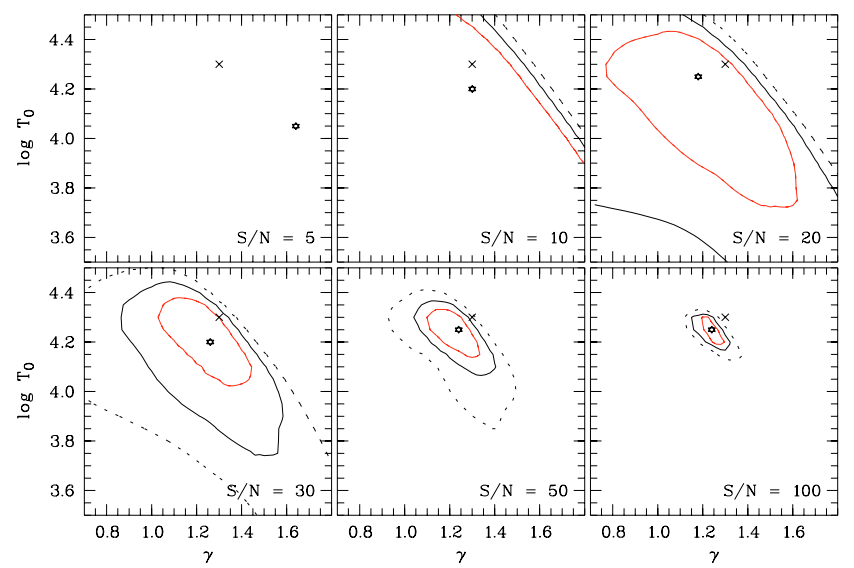

Fig. 1. Contour plots for the parameters of the temperature-density relation derived from the simulated data. The crosses represent the position of the true values of $\gamma=1.3$ and $\log T_{0}=4.3$ while the stars indicate the estimated minimum. The outer contours give the joint $1 \sigma$ (solid line) and $2 \sigma$ (dotted line) confidence levels, respectively. The inner contour represents the $1 \sigma$ confidence level for one free parameter, i.e. the projection of the inner contour to the axes leads to the $1 \sigma$ errors of $\log T_{0}$ and $\gamma$, respectively. The signal-to-noise ratio of the artificial He II spectra is indicated in each panel. The confidence levels in case of $S / N=5$ (upper left panel) are outside the presented parameter range.
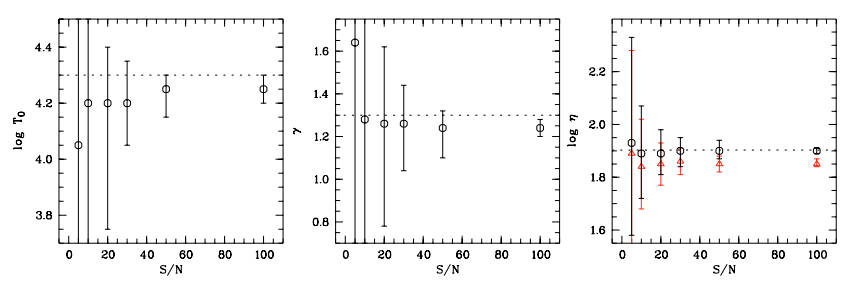

Fig. 2. Results for the fit of the artificial data. The panels show the derived values for $\log T_{0}, \gamma$, and $\log \eta$ (from the left to the right). The error bars correspond to the inner $1 \sigma$ confidence contours of Fig. 1 . The dotted lines indicate the true values. For comparison the $\eta$ values estimated under the assumption of pure turbulent line broadening are presented as triangles in the right panel.

level of the best optical spectra available today and is sufficient to derive a reasonable line list from the fit.

The H I Ly $\alpha$ forest is fitted with Doppler profiles once and afterwards all artificial He II spectra are fitted on the basis of the derived line list according to the method presented in Sect. 3. Figure 1 shows the $1 \sigma$ and $2 \sigma$ confidence contours for the parameters $\log T_{0}$ and $\gamma$ of the temperature-density relation (Eq. (1)). The point of the best fit is marked by a star, while the true underlying value is indicated by a cross. As expected, the results are better constrained if the $S / N$ of the He II spectrum is increased. In case of $S / N=5$ a reasonable estimate cannot be derived since the variation of the absorber's temperature affects the wings of the line profiles only marginally and due to the high noise level very similar $\chi^{2}$-values are produced. In order to derive reasonable constraints for the temperature-density relation a signal-to-noise ratio of at least $S / N \sim 20 \ldots 30$ is required for the He II data. The results are summarized in Fig. 2 which presents the best fit values and their $1 \sigma$ uncertainties in comparison to the true underlying values. It becomes clear that also for $S / N=100$ the derived $\log T_{0}$ and $\gamma$ are underestimated. The reason for this systematic effect is the imperfectness of the adopted line list (see below). However, the inferred $\eta$ values are in good agreement with the underlying $\eta=80$.

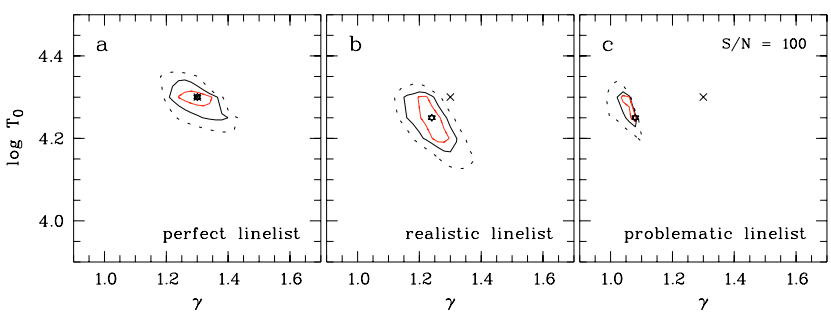

Fig. 3. Contour plots for the parameters of the temperature-density relation derived from the artificial data using three different $\mathrm{HI}$ line lists for the He II fitting (see text). The crosses represent the position of the true values of $\gamma=1.3$ and $\log T_{0}=4.3$ while the stars indicate the estimated minimum. Contour levels are given as in Fig. 1.

For comparison we also perform a fit assuming pure turbulent line widths, i.e. $b_{\mathrm{He} \text { II }}=b_{\mathrm{HI}}$. The resulting $\eta$ values are presented as triangles in the right panel of Fig. 2. Obviously, the $\eta$ value is underestimated if pure turbulent line broadening is assumed. This finding is essentially independent of the signal-to-noise ratio of the $\mathrm{He}$ II and also of the modifications of the line list (see below). On average the pure turbulent $\eta$ value is $\sim 0.05$ dex lower than the $\eta$ derived when considering a temperature-density relation and thus about 0.05 dex lower than the underlying value. This is still consistent within the $1 \sigma$ level in case of low $S / N$ but the underestimation will be significant as the error bars decrease for higher quality data $(S / N \gtrsim 50)$.

As noted above, the best fit depends on the quality of the adopted line list. This is illustrated in Fig. 3. We performed fits to the artificial He II spectrum with $S / N=100$ based on three different H I line lists. The "perfect" line list includes all absorption lines that have been generated to compute the artificial spectra. In this case the correct parameters are, of course, reproducible (Fig. 3a). We find $\log T_{0}=4.30 \pm 0.02$ and $\gamma=1.30_{-0.06}^{+0.04}$. For a "reasonable" line list based on a thorough fit of the H I spectrum we obtain $\log T_{0}=4.25 \pm 0.05$ and $\gamma=1.24 \pm 0.04$ (Fig. 3b). In particular $\gamma$ is not reproduced correctly although the estimated value has apparently small error bars. In this case the imperfectness of the line list introduces additional systematic uncertainties.

Furthermore, we examine a realistic but "problematic" case (Fig. 3c). The difference between the realistic and the problematic line list is illustrated in Fig. 4. A broad, saturated H I feature has been fitted with two Doppler profiles (realistic case) or with one component only (problematic case). The upper panel of Fig. 4 shows only very slight discrepancies between the resulting profiles. However, there are more prominent differences in the He II fit (lower panel of Fig. 4). As a consequence of the absence of a second component the feature has to be modeled by a broader profile to achieve a reasonable fit. Therefore, the temperature $T$ has to be lower according to Eq. (7). Because of the given H I column density of the line $\gamma$ has to decrease (see Eq. (6)). Indeed, we find $\log T_{0}=4.25_{-0.01}^{+0.05}$ and $\gamma=1.08_{-0.04}^{+0.01}$ (Fig. 3c). Additionally, a weak $\mathrm{H} \mathrm{I} \operatorname{line}\left(\log N_{\mathrm{HI}}=11.7\right)$ leading to a more prominent He II feature $\left(\log N_{\mathrm{He} \text { II }}=13.4\right)$ at $1001.0 \AA$ is missed in both models introducing further deviations from the optimum model.

However, in case of observed spectra the best strategy to produce the $\mathrm{H}$ I line list would also take into account higher order features of the Lyman series. Thus, the estimation of the line parameters of a saturated absorption complex would be more accurate than in this simple simulated case. Due to blending or incomplete spectral coverage a few features with ambiguous line parameters might still be left unsettled even for observed 

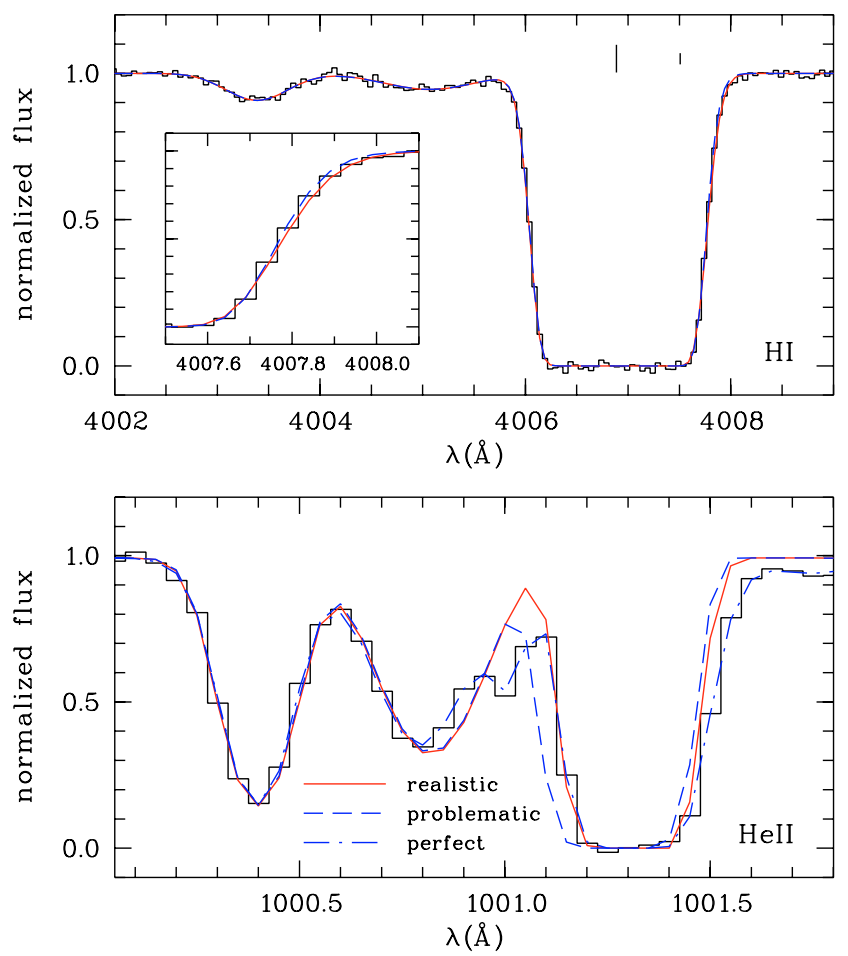

Fig. 4. Illustration of the importance of the HI fit. The upper panel shows a portion of the artificial H I data (histogram-like) and two different fits where the saturated feature at $4007 \AA$ is modelled with one (dashed line) or two (solid line) Doppler components, respectively. The inset in the upper panel illustrates the only marginal difference between both fits. The lower panel shows the artificial He II data (histogram-like) and the best fits in case of the one component approach (dashed line; $\log T_{0} \approx 4.25, \gamma \approx 1.08$ ) or two component fit (solid line; $\log T_{0} \approx 4.25$, $\gamma \approx 1.24$ ), respectively. The dot-dashed line represents the best fit using the perfect line list $\left(\log T_{0}=4.30, \gamma=1.30\right)$.

spectra. But the existence of unidentified, weak $\mathrm{H}$ I features below the detection limit can never be excluded. For this reason profile fit based analyses of the He II Ly $\alpha$ forest include He II lines without detected H I counterparts (Kriss et al. 2001; Zheng et al. 2004; Fechner et al. 2006a). One should keep in mind that even one missing component may affect the results significantly.

\section{Application to observations}

Though the data quality for the two He II quasars observed with FUSE, HE 2347-4342 and HS 1700+6416 $(S / N \sim 5)$, is well below the required noise level of $S / N \gtrsim 20$, we apply the method presented in Sect. 3 to the observed spectra. Our main intention is to explore whether any conclusions can be inferred even from this noisy data. In order to avoid additional degrees of freedom the scales to perform a fit are selected a priori. We select spectral ranges with a size of $\sim 5 \AA$, corresponding to $\Delta z \sim 0.016$. Scales of variation of the UV background in this order of magnitude have been estimated by Fechner \& Reimers (2007), whereas Shull et al. (2004) and Bolton et al. (2006) find much smaller scales in the range $\Delta z \sim 0.001$. Realistically, we do not expect to be able to derive robust constraints on the parameters of the temperature-density relation. However, this approach should be sufficient to roughly estimate the effect of partly thermal Doppler parameters on the results.

The far-UV data of HE 2347-4342 are presented in Zheng et al. (2004). We use the normalization described in

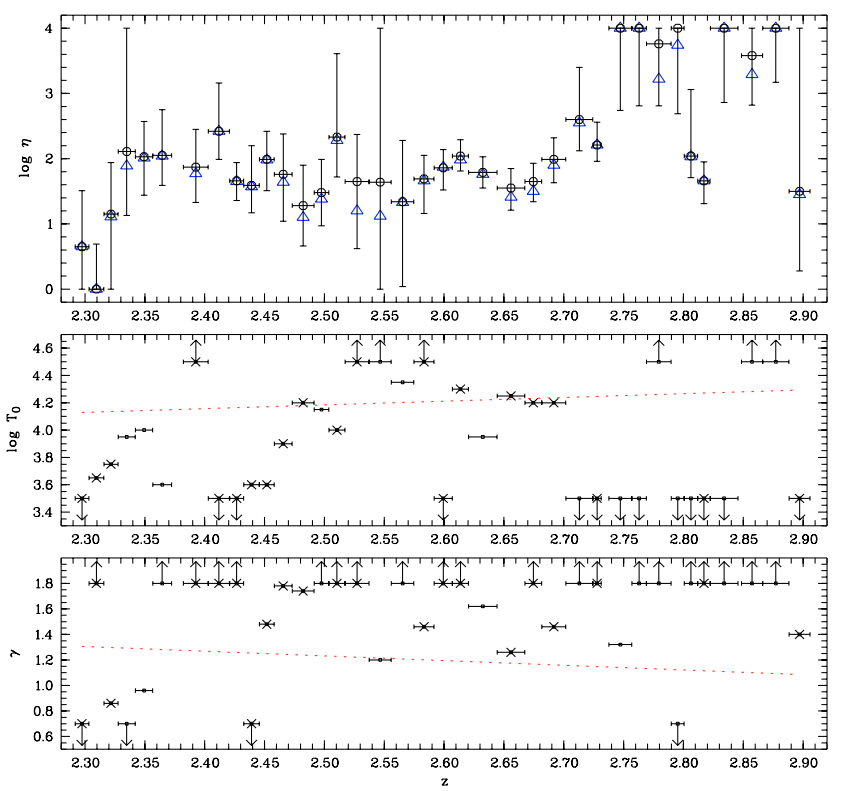

Fig. 5. Results from HE 2347-4342 for $\log \eta$ (open circles; upper panel), $\log T_{0}$ (middle panel), and $\gamma$ (lower panel). The triangles in the upper panel present the resulting $\eta$ value assuming pure turbulent broadening. For clarity error bars are not given for the turbulent case, but are of the same size as those of the temperature-density relation fit. The $1 \sigma$ uncertainties of $\log T_{0}$ and $\gamma$ would comprise the whole presented parameter range. Fit sections of $\sim 5 \AA$ have been selected a priori. Sections marked with a cross represent redshift intervals without extremely saturated absorption features. The dotted lines in the lower panels indicated the expected value interpolated from the measurements of Schaye et al. (2000).

Fechner \& Reimers (2007). The spectrum is binned to $0.05 \AA$ which corresponds to the resolution of $R=20000$ at $1000 \AA$ (and the actual value $\sim 15 \mathrm{~km} \mathrm{~s}^{-1}$ ) and reduces the noise to a $S / N \sim 5$ level per bin in the whole spectrum. In order to avoid further uncertainties we exclude the spectral range containing Ly $\beta$ or higher order Lyman series lines. For the H I Ly $\alpha$ forest towards HE 2347-4342 we adopt the line list of Fechner \& Reimers (2007) based on a Doppler profile fit of high-quality VLT/UVES spectra $(R \approx 45000, S / N \approx 100)$. We slightly modify the parameters of the Lyman limit system complex at $z \sim 2.735$ which are now estimated using the Lyman series up to Ly-9.

The FUSE data of HS $1700+6416$ are presented in Fechner et al. (2006a). It has a resolution of $R \sim 20000$. The signalto-noise ratio is $\sim 5$ and slightly better. The line list for the H I Ly $\alpha$ forest is adopted from the results of Fechner et al. (2006a) who use high-quality data from Keck/HIRES $(R \approx$ $38500, S / N \approx 100$ ). Furthermore, we correct for metal lines in the FUSE spectral range as described by Fechner \& Reimers (2007) using the models of Fechner et al. (2006b).

As a compromise between constructing a model as realistic as possible and the caveats due to the limited quality of the data we fit the selected spectral ranges assuming constant values of $\eta, \log T_{0}$, and $\gamma$. For comparison we also estimate a fit assuming pure turbulent line widths. The results are presented in Figs. 5 (HE 2347-4342) and 6 (HS 1700+6416). Towards HE $2347-4342$ the fit intervals at $z \gtrsim 2.75$ cover the range of patchy He II absorption (Reimers et al. 1997) which marks the tail end of the epoch of He II reionization (also e.g. Kriss et al. 2001; Zheng et al. 2004). For these redshift ranges further uncertainties like the incomplete reionization of He II (e.g. Reimers et al. 1997, 2005) or simply the fact that the FUSE spectrum 


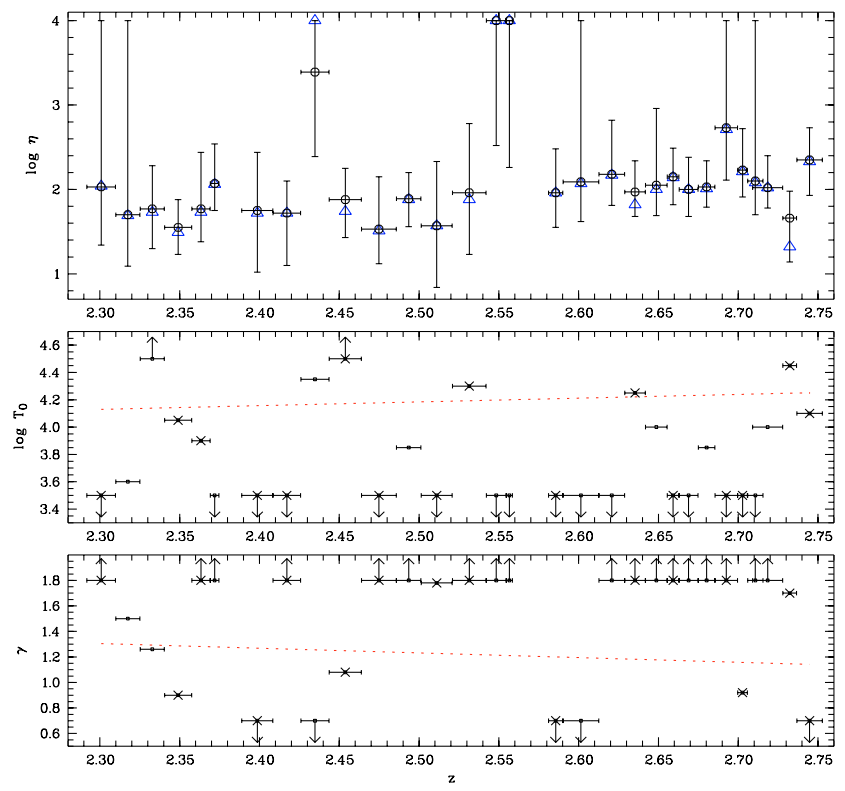

Fig. 6. As Fig. 5 but for HS 1700+6416.

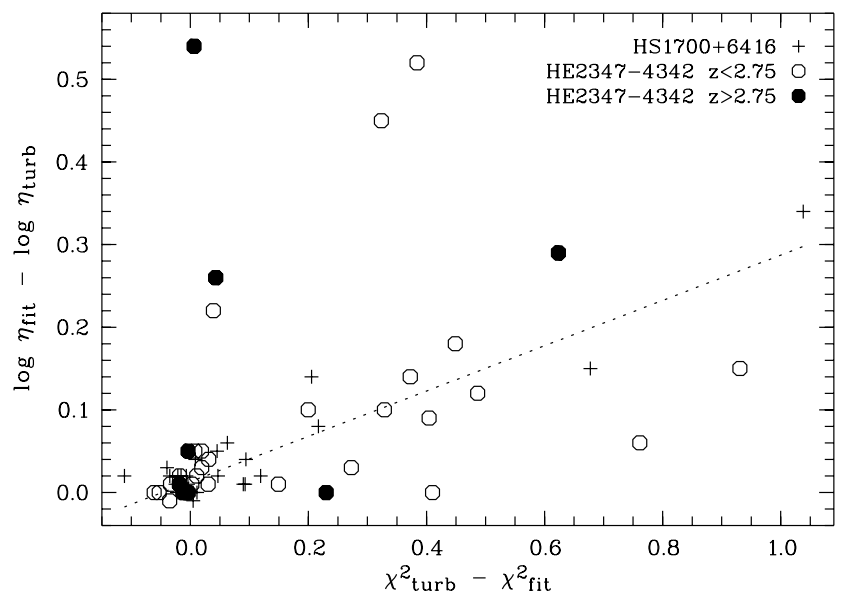

Fig. 7. Correlation between the difference in the quality of the fit, i.e. the $\chi^{2}$, and the difference in the $\eta$ value. The better the fit taking into account a temperature-density relation in comparison the the fit assuming pure turbulent broadening, the larger is the resulting $\log \eta$ for the best fit. The dotted line represents a linear fit to the $\operatorname{Ly} \alpha$ forest data, i.e. HS 1700+6416 and HE 2347-4342 at redshifts $z<2.75$.

shows completely saturated, broad absorption troughs, lead to extremely unstable fits which should not be considered further.

Generally, the $\eta$ values in the pure turbulent cases are slightly lower than if the thermal state of the IGM is taken into account, consistent with the results from the artificial data. On average the turbulent $\eta$ is lower by roughly 0.05 dex considering the Ly $\alpha$ forest only (i.e. $z<2.75$ ). The difference in the $\eta$ value is correlated with the quality of the fit, i.e. with the $\chi^{2}$, which is shown in Fig. 7. If a thermal component to the line width is considered, the He II lines become narrower in comparison to the pure turbulent case. In order to produce roughly the same equivalent width the column density of He II and thus $\eta$ has to increase. The correlation presented in Fig. 7 means if considering the thermal state of the absorbers significantly improves the fit in a selected He II wavelength range, the inferred $\eta$ value will be larger. And even if the fit is improved only slightly $\left(\chi_{\text {turb }}^{2}-\chi_{\text {fit }}^{2}<0.1\right)$, the $\eta$ value increases on average by $0.02 \mathrm{dex}$ (median $0.01 \mathrm{dex}$ ).

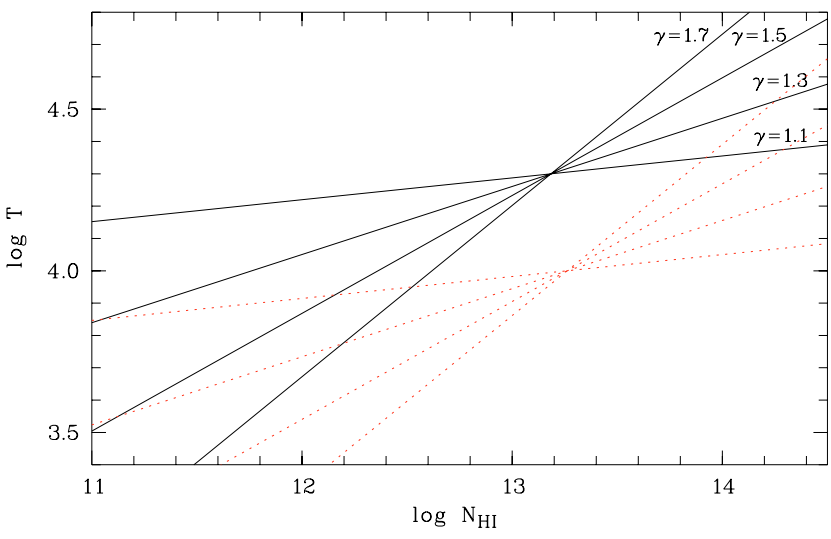

Fig. 8. Relation between $\mathrm{H}$ I column density $\log N_{\mathrm{HI}}$ and the absorber's temperature $\log T$ according to Eq. (6). The relations are shown for $\log T_{0}=4.3$ (solid lines) and $\log T_{0}=4.0$ (dotted lines) and $\gamma=1.7$, 1.5, 1.3, and 1.1.

However, the deviations are still within the $1 \sigma$ uncertainties of the estimate (see also the upper panels of Figs. 5 and 6).

The parameters of the temperature-density relation estimated by the fits are rather unconstrained. As described in Sect. 3 we explored the parameter space $3.50 \leq \log T_{0} \leq 4.50$ and $0.70 \leq$ $\gamma \leq 1.80$. Within these limits all values produce models fitting to the data on a $1 \sigma$ level. Therefore, no error bars are presented in Figs. 5 and 6. Nevertheless, some tendencies are noteworthy.

The vast majority of the fit intervals exhibit an extreme value for $\gamma(\leq 0.7$ or $\geq 1.8)$ and/or $\log T_{0}(\leq 3.5$ or $\geq 4.5)$. Excluding the redshift range with patchy He II absorption $(z \gtrsim 2.75)$ from further discussion, $81 \%$ of the fitting sections yield extreme values of the temperature-density relation. Among these cases $34 \%$ lead to a temperature-density relation with $\gamma \geq 1.8$ and $\log T_{0} \leq 3.5$.

In order to give an interpretation of the temperature-density relation in this case some examples are illustrated in Fig. 8. A large value of $\gamma$ leads to a steep relation between column density and temperature. Consequently, the temperature of high column density absorbers is much higher than the temperature of low column density absorbers. The parameter $\log T_{0}$ rules the absolute values of the temperature. The $\operatorname{lower} \log T_{0}$, the lower is the general temperature level. Finding $\gamma \geq 1.8$ means that the line widths $b_{\mathrm{HeII}}$ of high column density absorbers are significantly narrower than $b_{\mathrm{HI}}$. Therefore thermal line broadening is favored. This idea may support the conclusions of Fechner \& Reimers (2007) who suggest that thermal line broadening might be important for high column density absorbers.

For about $9 \%$ of the extreme cases we find $\gamma \leq 0.70$ and $\log T_{0} \leq 3.50$, the lowest values in the parameter range explored. At this point the lowest temperatures are produced for high column density absorbers since the slope $(\gamma-1)$ of the temperaturedensity relation gets negative and $\log T_{0}=3.5$ sets the absolute scale to low temperatures. Thus, thermal broadening might play a minor role for the line widths of the $\mathrm{H} \mathrm{I}$ and $\mathrm{He}$ II features. For these fit sections the $\eta$ values estimated from the pure turbulent and the thermal state model are nearly identical supporting the idea that turbulent line broadening is dominating in these redshift ranges. At $z \sim 2.43$ towards HS $1700+6416$ both derived $\eta$ values are heavily overproduced due to problems with two saturated, broad absorption features that dominate the selected spectral portion.

The results are expected to be more stable if regions with saturated or extremely weak $\mathrm{H}$ I are excluded. The data points marked with a cross in the lower panels of Figs. 5 and 6 represent 

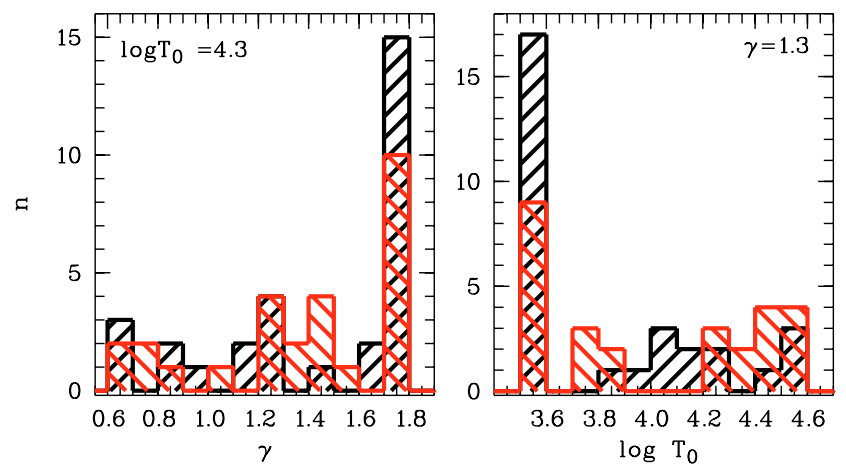

Fig. 9. Distribution of the parameters of the temperature-density relation estimated individually for the sight lines towards HS 1700+6416 (black) and HE 2347-4342 (light; $z<2.75$ ). The value of $\log T_{0}=4.3$ has been fixed when estimating $\gamma$ (left panel), while $\gamma=1.3$ when $\log T_{0}$ has been optimized (right panel).

the sections free from strongly saturated features. However, the results presented above for the whole spectra is also valid when considering only the selected portion of the data. Differences should be expected for data of higher quality.

Furthermore, we test to constrain the parameters of the temperature-density relation by fixing one parameter at a reasonable value and varying the other one and $\log \eta$ simultaneously. The estimated distributions of $\log \eta$ are very similar to those presented in Figs. 5 and 6. The rms-deviation from the distributions shown is $\sim 0.15$ or even smaller. Fixing $\log T_{0}=4.3$ and varying $\gamma$ still leads to inconclusive estimates of $\gamma$ for both lines of sight. The derived distributions are presented in the left panel of Fig. 9. The majority of redshift bins prefers an extreme value $\gamma \geq 1.8$ like in the standard procedure. Furthermore, the distribution might peak at $\gamma \sim 1.2$, which would be consistent with independent estimates from the literature (see below). However, the detection is insignificant for the present data. Similar results are found if $\gamma=1.3$ is fixed. The estimated distributions of $\log T_{0}$ are shown in the right panel of Fig. 9. In this case low values of $\log T_{0}$ are preferred like in Figs. 5 and 6 . There might be a slight tendency for $\log T_{0} \sim 4.4$ considering the HE 23474342 line of sight, while towards HS $1700+6416 \log T_{0} \sim 4.1$. However, these results are rather inconclusive. In order to give more significant constraints, He II data of better quality is needed or at least a more reliable statistics based on a larger sample.

In the literature Bryan \& Machacek (2000), Ricotti et al. (2000), Schaye et al. (2000), and McDonald et al. (2001) measured the parameters of the equation of state in the considered redshift range $(2.3<z<2.9)$ estimating the lower cut-off of the $b\left(N_{\mathrm{HI}}\right)$ distribution. Their resulting values are in the range $0.8 \lesssim \gamma \lesssim 1.7$ and $4.05 \lesssim \log T_{0} \lesssim 4.45$ with a strong redshift dependence. Because of the just completed epoch of He II reionization $\gamma$ increases steeply towards lower redshifts while $\log T_{0}$ decreases with $z$. Fitting a straight line to the results of Schaye et al. (2000) in the redshift range $1.8<z<3.2$ we find $\gamma=-(0.37 \pm 0.16) \cdot z+(2.15 \pm 0.42)$ and $\log T_{0}=$ $(0.27 \pm 0.06) \cdot z+(3.50 \pm 0.15)$, respectively. The results of Schaye et al. (2000) are selected since they cover a large redshift range including the portion we are interested in. The other authors use slightly different methods and provide fewer data points (difference between the methods and systematic offsets in the results are discussed in Bryan \& Machacek 2000; Ricotti et al. 2000; McDonald et al. 2001). For comparison the fit to the Schaye et al. (2000) data is indicated in Figs. 5 and 6 as dotted lines. It is obvious that our results scatter over the range given by the literature and beyond. Furthermore, there is no significant concentration of measurements towards the Schaye et al. (2000) interpolation. Therefore, a detailed comparison is meaningless. As demonstrated in Sect. 4 UV data of higher quality $(S / N \gtrsim 20)$ would be needed to derive tighter constraints for the temperature-density relation using the He II Ly $\alpha$ forest.

In addition, recent indications for a small value of $\gamma \approx 1.1$ found by D'Odorico et al. (2006) investigating the clustering properties in a sample of close QSO pairs, or even hints of an inverses temperature-density relation $(\gamma \approx 0.5)$ which would improve a fit of the observed Ly $\alpha$ flux probability distribution function of a sample of 63 quasars reported by Becker et al. (2006) cannot be addressed here due to the limited data quality of the He II spectra.

\section{Direct temperature estimate}

Even though we showed in the previous sections that an estimate of the IGM temperature requires better UV data, we also follow an alternative approach to estimate the temperatures of the absorbers more directly. An advantage of this alternative procedure is that the scale of the UV background variations can be estimated. Therefore, the method described in Sect. 3 is adjusted. Instead of the temperature-density relation the temperature of each line itself is fitted by varying the Doppler parameter of $\mathrm{He}$ II according to Eq. (7). We start with the pure turbulent case $b_{\mathrm{He} \text { II }}=b_{\mathrm{HI}}$ and then change the thermal component. Furthermore, a scale estimation as described in Fechner \& Reimers (2007) is implemented.

A fit is computed for a given size of an interval. Then the bin size is increased until the $\chi^{2}$ of the best fit gets worse again. While the spectrum fit method uses the observed optical data directly and may therefore facilitate a pixelwise increment of the fit interval, the approach presented here is based on a superposition of Doppler profiles. Therefore it has to be ensured that all components possibly contributing at a certain wavelength are taken into account. Experimenting with several strategies in test calculations we choose a starting interval of $0.5 \AA$ in He II which is increased by 3 pixels, i.e. $0.15 \AA$, per iteration. Furthermore, if the enlargement of the fit interval leads to a larger $\chi^{2}$, the interval is increased (up to 5 times) to test whether a better fit can be obtained.

The best fit for the redshift range $2.58<z<2.74$ towards HE 2347-4342 is presented in Fig. 10. The corresponding fit of the spectrum of HS $1700+6416$ is shown in Fig. 11. The redshift range $2.58<z<2.74$ is chosen since the quality of the FUSE data in the corresponding spectral range is best for both lines of sight. In the following we will discuss the results for this regime.

As can be seen from the lower panels of Figs. 10 and 11, the estimated temperature is only poorly constrained. Temperatures can be estimated for about $55 \%$ of the lines (presented as circles). However, in most of the cases $(65.5 \%)$ the $1 \sigma$ uncertainties cover the whole range from the high temperature cut-off, when $b_{\mathrm{H} \text { I }}$ is interpreted as completely thermal, to the pure turbulent limit. On the other hand, $45 \%$ of the lines favor turbulent line broadening or yield a very low temperature (we set the minimum temperature to $10 \mathrm{~K}$ corresponding to a thermal line width $<0.5 \mathrm{~km} \mathrm{~s}^{-1}$ which can be interpreted as purely turbulent). The upper limits estimated for those absorbers are also indicated in Figs. 10 and 11. In total only 6 lines $(\sim 2 \%)$ lead to well-constrained temperature estimates. All of them favor the purely thermal interpretation of the H I Doppler parameter. The resulting distribution of the line temperatures is shown in the left 


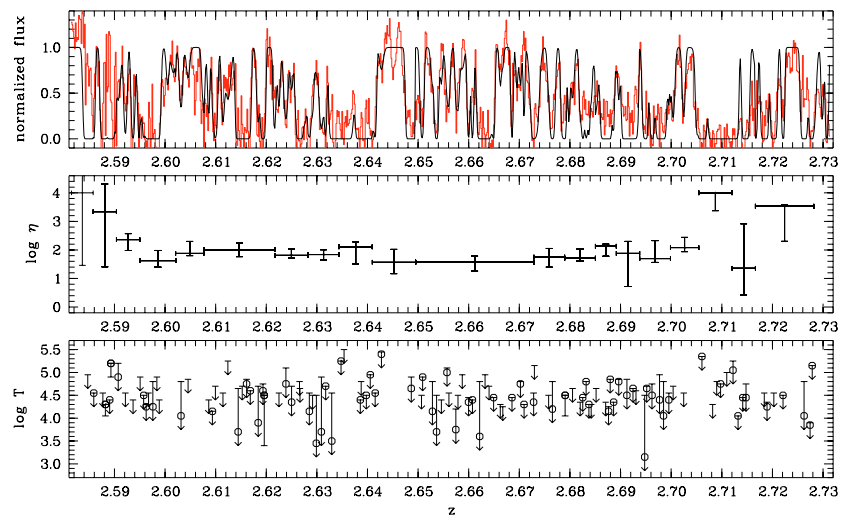

Fig. 10. Best fit towards HE 2347-4342 in the redshift range $2.58 \leq$ $z \leq 2.73$ (upper panel). Fitted parameters are the temperatures of the absorbers (lower panel), the He II/H I column density ratio $\eta$ (middle panel), and the scale of $\eta$ variation. The resulting temperatures are presented as open circles in the lower panel. However, most of them have large error bars and are actually upper limits. No temperature is given if turbulent line broadening is preferred (see also text). At $z \sim 2.69$ two saturated Hi lines appear to be stronger than the corresponding He II features in the line center leading to a poor fit in this range.

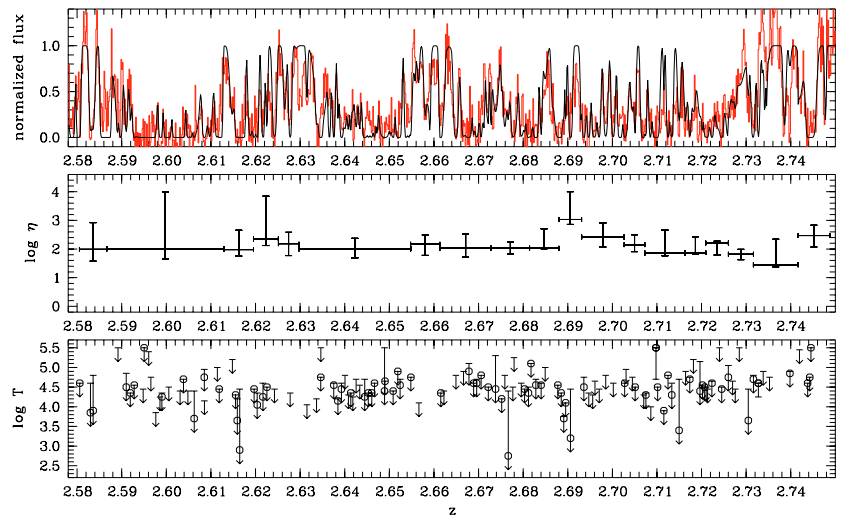

Fig. 11. Best fit and resulting parameters towards HS $1700+6416$ in the redshift range $2.58<z<2.75$; lines and symbols as in Fig. 10 .

panel of Fig. 12, excluding components that prefer turbulent line widths. The average value is $\log T=4.41 \pm 0.46$.

In order to obtain an estimate of the equation of state the derived temperature is plotted versus the H I column density in Fig. 13. Due to the large confidence intervals of the temperature only a slight correlation between the $\log N_{\mathrm{H} \text { I }}$ and $\log T$ can be seen. The Spearman rank-order correlation coefficient is $r_{\mathrm{s}}=0.22$. Consequently, a linear fit to the data according to Eq. (6) is rather meaningless. The dotted line in Fig. 13 indicates a temperature-density relation with the parameters $\gamma=1.17$ and $\log T_{0}=4.23$ as extrapolated from the results of Schaye et al. (2000, see previous section). Our temperature estimates have huge error bars (the majority of points presented in Fig. 13 are in fact upper limits), but the relation derived from the Schaye et al. (2000) results is not inconsistent with our measurements. Harder constraints of the line temperature are needed to improve the results. Also the direct temperature estimate would benefit from higher $S / N$ data of the He II Ly $\alpha$ forest.

Another point is the determination of the column density ratio $\eta$ and its variation with redshift for the pure turbulent broadening versus the thermal plus turbulent broadening cases. The right panels of Fig. 12 show the distribution of the resulting $\eta$ values and the scales of its variation. The average $\mathrm{He} \mathrm{II} / \mathrm{H}$ I

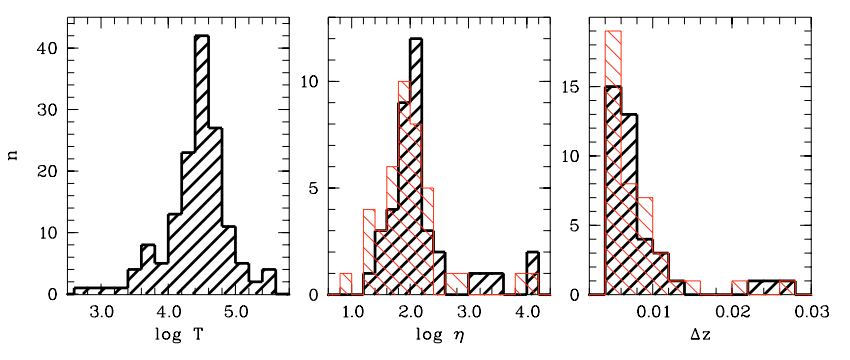

Fig. 12. Distribution of the estimated parameters in the redshift range $2.58<z<2.74$ towards HE 2347-4342 and HS 1700+6416. Presented are the distributions of the line temperature $\log T$ (left panel), the He II/H I column density ratio $\log \eta$ per scale (middle panel, thick line), and the scale size $\Delta z$ of $\eta$ variations (right panel, thick line). The thinlined histograms in the right panels indicate the distributions of $\log \eta$ and $\Delta z$, respectively, in the case of turbulent line widths $b_{\mathrm{He} \mathrm{II}}=b_{\mathrm{HI}}$.

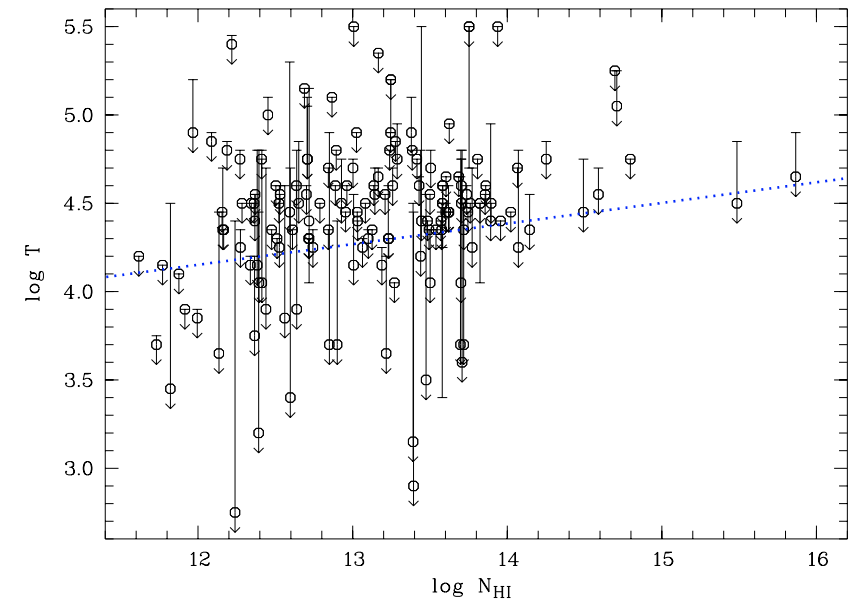

Fig. 13. Line temperature $\log T$ versus $\mathrm{HI}_{\mathrm{I}}$ column density $\log N_{\mathrm{H}}$ for the combined sample of HE 2347-4342 and HS 1700+6416 in the redshift range $2.58<z<2.74$. Only lines that lead to realistic best-fit temperatures are indicated. The mean redshift of the considered line sample is $z=2.663 \pm 0.082$. The dotted line indicated a temperaturedensity relation with $\gamma=1.17$ and $\log T_{0}=4.23$ as interpolated from the results of Schaye et al. (2000).

ratio for the combined sample is $\log \eta=2.16 \pm 0.62$ (median 2.01), where the line of sight towards HE 2347-4342 contributes most of the scatter. Towards this QSO we find the mean value $\log \eta=2.21 \pm 0.82$ (median 1.88; note the outliers in Fig. 10) while HS $1700+6416$ yields $\log \eta=2.11 \pm 0.32$ (median 2.03). Also shown in Fig. 12 is the distribution of $\eta$ values for the case of fixed $b_{\mathrm{He} \text { II }}=b_{\mathrm{HI}}$ Doppler parameters. The pure turbulent fits yield roughly $0.1-0.2$ dex lower $\eta$ values. For the combined sample we find the mean $\log \eta=1.98 \pm 0.59$ (median 1.90). The results for the individual sight lines are $\log \eta=2.00 \pm 0.63$ (median 1.85) towards HE 2347-4342 and $\log \eta=1.97 \pm 0.58$ (median 1.95) towards HS 1700+6416. Furthermore, the distribution of $\eta$ values is broader in comparison to the thermal fit result. Fitting a Gaussian to the $\log \eta$ distributions shown in the middle panel of Fig. 12 yields a FWHM of $0.67 \pm 0.12$ for the temperature fit but $0.98 \pm 0.14$ in case of pure turbulent broadening. An explanation for this finding may be the correlation of $\eta$ with the H I column density due to the assumption of pure turbulent broadening which we will discuss in the following. If the estimate of $\eta$ is on average too low for high density absorbers, the distribution of $\eta$ values will become broader and shifted to lower values. 
One of the main conclusion of Fechner \& Reimers (2007) has been that the assumption of pure turbulent line broadening may lead to the observed correlation between the strength of the $\mathrm{HI}$ absorption and $\eta$ (e.g. Shull et al. 2004). Comparing the results of the fits considering the line temperature or assuming turbulent line widths, we find indications that this correlation is less pronounced if thermal line broadening is taken into account. The average $\eta$ value for $N_{\mathrm{HI}}>10^{13} \mathrm{~cm}^{-2}$ absorbers is $\log \eta=2.02 \pm 0.55$ (median 2.01) for the thermal fit and $\log \eta=$ $1.83 \pm 0.36$ (median 1.87) in the turbulent case. If $b_{\mathrm{He} \mathrm{II}}=b_{\mathrm{HI}}$ is assumed, the median $\eta$ value clearly depends on the H I column density of the absorber, while for $12.0<\log N_{\mathrm{HI}}<13.0$ the median $\log \eta$ is 1.93 , it is lower by 0.06 dex for high column density lines $\left(\log N_{\mathrm{HI}}>13.0\right)$. On the other hand the mean $\eta$ value is independent of H I column density if thermal broadening is considered. In this case the median is 2.01 in both column density ranges.

The size of the scale of $\eta$ variations is comparable towards both quasars. The scales are in the range $0.0041 \leq \Delta z \leq 0.0263$ (mean $\Delta z=0.0081 \pm 0.0054$, median 0.0061) corresponding to $\sim 3-4 h^{-1} \mathrm{Mpc}$ up to $20 h^{-1} \mathrm{Mpc}$ comoving, which is slightly less than the scales found by Fechner \& Reimers (2007) using the original spectrum fitting method. As also can be seen from the right panel of Fig. 12, we find no significant deviation for the distribution of scale sizes derived under the assumption of pure turbulent line broadening or thermal plus turbulent broadening.

\section{Summary and conclusions}

We have introduced an approach to take into account the thermal state of the IGM when analyzing the He II Ly $\alpha$ forest which has been resolved towards the quasars HE 2347-4342 and HS $1700+6416$. The intention of this procedure is twofold. At first, systematic errors due to the assumption of pure turbulent line broadening in the analysis of the He II Ly $\alpha$ forest should be eliminated. Following Fechner \& Reimers (2007) the He II/H I column density ratio $\eta$ may be underestimated in case of strong absorbers if turbulent line widths are assumed. This may lead to a spurious correlation of strength of the absorber with the $\eta$ value. Secondly, investigating the combined He II and H I Ly $\alpha$ forest could provide in principle an independent strategy to estimate the temperature and the thermal state of the IGM.

The procedure is based on the spectrum fitting method introduced by Fechner \& Reimers (2007). Instead of the observed optical data a spectrum computed from the Doppler profile-fitted $\mathrm{HI}$ line list is fitted to the He II data. Using Doppler profiles enables us to modify the temperature of the absorber as an additional parameter. Consequently, three parameters are estimated, $\gamma$ and $\log T_{0}$ of the temperature-density relation as well as the column density ratio $\eta$.

Artificial data are used to test the procedure. We find that a minimum quality of the He II data, $S / N \gtrsim 20$, is needed to derive reasonable constraints of IGM's thermal state. Furthermore, the $\mathrm{H}$ I line list may severely influence the results. Ambiguities in the decomposition of blends can introduce systematic errors to the resulting fit and even one missing component may prevent the procedure from recovering the true temperature-density relation.

Applying the procedure to the observed spectra of the quasars HE 2347-4342 and HS 1700+6416 leaves the parameters of the temperature-density relation unconstrained due to the low signal-to-noise ratio of the He II data $(S / N \sim 5)$. However, some of the fitted redshift ranges show indications of favoring either pure thermal or pure turbulent line broadening.
Table 1. Summary of $\eta$ values derived with the method of direct temperature estimates in the redshift range $2.58 \leq z \leq 2.74$.

\begin{tabular}{lcccc}
\hline \hline & \multicolumn{2}{c}{ Turbulent } & \multicolumn{2}{c}{ Thermal + turbulent } \\
& $\langle\log \eta\rangle$ & median & $\langle\log \eta\rangle$ & median \\
\hline HE 2347-4342 & $2.00 \pm 0.63$ & 1.85 & $2.21 \pm 0.82$ & 1.88 \\
HS 1700+6416 & $1.97 \pm 0.58$ & 1.95 & $2.11 \pm 0.32$ & 2.03 \\
both sight lines & $1.98 \pm 0.59$ & 1.90 & $2.16 \pm 0.62$ & 2.01 \\
\hline
\end{tabular}

Taking into account in addition thermal broadening leads to different line widths for $\mathrm{HI}$ and He I with $b_{\mathrm{He} \text { II }}<b_{\mathrm{HI}}$. Therefore, the He II column density resulting from the fit considering a combination of thermal and turbulent broadening is systematically increased in comparison to a fit assuming the pure turbulent case, i.e. $b_{\mathrm{He} \text { II }}=b_{\mathrm{HI}}$. We find a systematic offset of roughly 0.05 dex which is within the $1 \sigma$ uncertainties of the estimates. The lower the $\chi^{2}$ of the fit including the temperaturedensity relation in comparison to the $\chi^{2}$ of the pure turbulent fit, the higher is the $\eta$ value. Considering the combined sample of selected fit intervals towards HS 1700+6416 and HE 2347-4342 the median $\log \eta=1.96$ is obtained from the thermal state fit, slightly higher than the median value 1.93 found by Fechner $\&$ Reimers (2007) considering only lines with $N_{\mathrm{HI}}<10^{13.0} \mathrm{~cm}^{-2}$, whereas the turbulent fit leads to 1.88 .

Alternatively, we estimate the thermal line width of the individual absorption features more directly by optimizing the Doppler parameter of the He II lines. In practice the values of the line temperature, the $\eta$ value, and the scale of the variation of $\eta$ are fitted simultaneously. We find that $45 \%$ of the lines favor turbulent broadening. The inferred temperatures of the remaining $55 \%$ of the components suffer from large uncertainties. Thus, it is impossible to derive a temperature-density relation. However, comparing the fit which includes a temperature optimization, to a fit assuming pure turbulent line broadening confirms the suspicion of Fechner \& Reimers (2007) that neglecting thermal line widths leads to a correlation of the inferred $\eta$ value with H I column density. While the pure turbulent fit results in lower $\eta$ values for high column density absorbers (median $\log \eta=1.87$ for lines with $\log N_{\mathrm{HI}}>13.0$ in comparison to 1.93 for lines with $\left.12.0<\log N_{\mathrm{H} \mathrm{I}}<13.0\right)$, the thermal fit yields no correlation at all (median $\log \eta=2.01$ for both column density ranges). This effect is also noticeable when comparing the distribution of $\eta$ values since a narrower distribution by $46 \%$ is found if a temperature is taken into account.

The inferred scales of $\eta$ variations are roughly $4 h^{-1} \mathrm{Mpc}$ comoving. But also large scales up to $\Delta z \sim 0.03$, corresponding to $\sim 20 \mathrm{~h}^{-1} \mathrm{Mpc}$ comoving, have been inferred. The derived scales appear to be independent of the assumption of the dominating broadening mechanism.

Thus, we confirm the existence of a thermal component which results in narrower He II features in comparison to $\mathrm{HI}$, affects the inferred $\eta$ value. An increased He II/H I ratio is found if a thermal component is taken into account. At redshifts $2.58<z<2.74$ we find $\eta \approx 100$ in agreement the value found by Fechner et al. (2006a) at the same redshifts towards HS $1700+6414$ alone (a summary of the derived $\eta$ values using the method presented in Sect. 6 is given in Table 1). However, constraining the parameters of the temperature-density relation and thereby providing an independent estimate of the thermal state of the IGM is impossible with the present day quality of $\mathrm{He}$ II Ly $\alpha$ forest observations. In order to obtain significant constraints a signal-to-noise ratio of $S / N \gtrsim 20$ would be 
required which is 4 times higher than the $S / N$ of the data available nowadays.

Acknowledgements. This work has been supported by the Deutsche Forschungsgemeinschaft (DFG) under RE 353/49-1.

\section{References}

Becker, G. D., Rauch, M., \& Sargent, W. L. W. 2006, ApJ, submitted [arXiv: astro-ph/0607633]

Bolton, J., Meiksin, A., \& White, M. 2004, MNRAS, 348, L43

Bolton, J. S., Haehnelt, M. G., Viel, M., \& Carswell, R. F. 2006, MNRAS, 366, 1378

Bryan, G. L., \& Machacek, M. E. 2000, ApJ, 534, 57

Burles, S., Nollett, K. M., \& Turner, M. S. 2001, ApJ, 552, L1

Cen, R., \& Ostriker, J. P. 1999, ApJ, 514, 1

Davé, R., Hernquist, L., Katz, N., \& Weinberg, D. H. 1999, ApJ, 511, 521

Davé, R., Cen, R., Ostriker, J. P., et al. 2001, ApJ, 552, 473

D’Odorico, V., Viel, M., Saitta, F., et al. 2006, MNRAS, 372, 1333

Fechner, C., \& Reimers, D. 2007, A\&A, 461, 847

Fechner, C., Reimers, D., Kriss, G. A., et al. 2006a, A\&A, 455, 91

Fechner, C., Reimers, D., Songaila, A., et al. 2006b, A\&A, 455, 73

Gleser, L., Nusser, A., Benson, A. J., Ohno, H., \& Sugiyama, N. 2005, MNRAS, 361, 1399

Hu, E. M., Kim, T., Cowie, L. L., Songaila, A., \& Rauch, M. 1995, AJ, 110, 1526

Hui, L., \& Gnedin, N. Y. 1997, MNRAS, 292, 27

Hui, L., \& Rutledge, R. E. 1999, ApJ, 517, 541
Kang, H., Ryu, D., Cen, R., \& Song, D. 2005, ApJ, 620, 21

Kirkman, D., \& Tytler, D. 1997, ApJ, 484, 672

Kriss, G. A., Shull, J. M., Oegerle, W., et al. 2001, Science, 293, 1112

Liu, J., Jamkhedkar, P., Zheng, W., Feng, L.-L., \& Fang, L.-Z. 2006, ApJ, 645, 861

Maselli, A., \& Ferrara, A. 2005, MNRAS, 364, 1429

McDonald, P., Miralda-Escudé, J., Rauch, M., et al. 2001, ApJ, 562, 52

Misawa, T., Tytler, D., Iye, M., et al. 2004, AJ, 128, 2954

Rauch, M., Becker, G. D., Viel, M., et al. 2005, ApJ, 632, 58

Reimers, D., Fechner, C., Hagen, H.-J., et al. 2005, A\&A, 442, 63

Reimers, D., Fechner, C., Kriss, G., et al. 2006, in ASP Conf. Ser., ed.

G. Sonneborn, H. W. Moos, \& B.-G. Andersson, 41

[arXiv: astro-ph/0410588]

Reimers, D., Köhler, S., Wisotzki, L., et al. 1997, A\&A, 327, 890

Ricotti, M., Gnedin, N. Y., \& Shull, J. M. 2000, ApJ, 534, 41

Schaye, J. 2001, ApJ, 559, 507

Schaye, J., Theuns, T., Leonard, A., \& Efstathiou, G. 1999, MNRAS, 310, 57

Schaye, J., Theuns, T., Rauch, M., Efstathiou, G., \& Sargent, W. L. W. 2000, MNRAS, 318, 817

Shull, J. M., Tumlinson, J., Giroux, M. L., Kriss, G. A., \& Reimers, D. 2004, ApJ, 600, 570

Theuns, T., Schaye, J., Zaroubi, S., et al. 2002a, ApJ, 567, L103

Theuns, T., Zaroubi, S., Kim, T.-S., Tzanavaris, P., \& Carswell, R. F. 2002b, MNRAS, 332, 367

Tittley, E. R., \& Meiksin, A. 2006, MNRAS, submitted [arXiv: astro-ph/0605317]

Valageas, P., Schaeffer, R., \& Silk, J. 2002, A\&A, 388, 741

Zheng, W., Kriss, G. A., Deharveng, J.-M., et al. 2004, ApJ, 605, 631 